\title{
Fuzzy quotient-3 cordial labeling of star related graphs- Paper I
}

\author{
P. Sumathi ${ }^{1 *}$ and J. Suresh Kumar $^{2}$
}

\begin{abstract}
Let $G(V, E)$ be a simple, finite and planar graph of order $p$ and size $q$. In this paper, the concept of Fuzzy Quotient-3 Cordial Labeling was introduced. Let $\sigma: V(G) \rightarrow[0,1]$ be a function defined by $\sigma(v)=\frac{r}{10}, r \in Z_{4}-\{0\}$. For each edge $u v$ define $\mu: E(G) \rightarrow[0,1]$ by $\mu(u v)=\frac{1}{10}\left\lceil\frac{3 \sigma(u)}{\sigma(v)}\right\rceil$ where $\sigma(u) \leq \sigma(v)$. The function $\sigma$ is called fuzzy quotient-3 cordial labeling of $G$ if the number of vertices labeled with $i$ and the number of vertices labeled with $j$ differ by at most 1 , the number of edges labeled with $i$ and the number of edges labeled with $j$ differ by at most 1 where $i, j \in\left\{\frac{r}{10}, r \in Z_{4}-\{0\}\right\}, i \neq j$. The number of vertices having label $i$ denotes $v_{\sigma}(i)$ and the number of edges having label $i$ denotes $e_{\mu}(i)$. Here it is proved that the Star graph and Star related graphs are Fuzzy Quotient-3 Cordial.
\end{abstract}

\section{Keywords}

Star, Cycle, Fuzzy quotient-3 cordial graph.

\section{AMS Subject Classification}

05C15.

${ }^{1}$ Department of Mathematics, C. Kandaswami Naidu College for Men, Chennai-600102, India.

${ }^{2}$ Department of Mathematics, St. Thomas College of Arts and Science, Chennai-600107, India.

*Corresponding author: ${ }^{1}$ sumathipaul@yahoo.co.in; ${ }^{2}$ jskumar.robo@gmail.com

Article History: Received 21 December 2018; Accepted 11 February 2019

\section{Contents}

1 Introduction 79

2

3

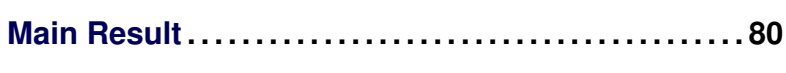

Conclusion . .82

References .82

\section{Introduction}

Graphs considered here are finite and simple. Graph labeling is used in several areas of science and technology like coding theory, astronomy, circuit design etc. The cordial labeling concept was first introduced by cahit [2]. The quotient3 cordial labeling have been introduced by $\mathrm{P}$. Sumathi, et al. found in [3-6]. They found some family of graphs are quotient-3 cordial. Motivated by these labelings we introduce fuzzy quotient-3 cordial labeling of graphs. If $G$ admits fuzzy quotient- 3 cordial labeling then $G$ is called as fuzzy quotient-3 cordial graph.

Definition 1.1. Let $\sigma: V(G) \rightarrow[0,1]$ be a function defined by $\sigma(v)=\frac{r}{10}, r \in Z_{4}-\{0\}$. For each edge uv define $\mu: E(G) \rightarrow$
$[0,1]$ by $\mu(u v)=\frac{1}{10}\left\lceil\frac{3 \sigma(u)}{\sigma(v)}\right\rceil$ where $\sigma(u) \leq \sigma(v)$. The function $\sigma$ is called fuzzy quotient-3 cordial labeling of $G$ if the number of vertices labeled with $i$ and the number of vertices labeled with $j$ differ by at most 1 , the number of edges labeled with $i$ and the number of edges labeled with $j$ differ by at most 1 where $i, j \in\left\{\frac{r}{10}, r \in Z_{4}-\{0\}\right\}, i \neq j$. The number of vertices having label $i$ denotes $v_{\sigma}(i)$ and the number of edges having label $i$ denotes $e_{\mu}(i)$.

Definition 1.2. A complete bipartite graph $K_{1, n}$ is said to be a star graph and it is denoted by $G_{1, n}$.

Definition $1.3\left(C_{3}(n)\right.$ star graph). The graph obtained by attaching $n$ pendent vertices in any one vertex of $C_{3}$ is called $C_{3}(n)$ star graph.

Definition $1.4\left(C_{3}(n, m)\right.$ star graph). The graph obtained by attaching $n$ pendent vertices in one vertex of $C_{3}, m$ pendent vertices in other one vertex of $C_{3}$ is called $C_{3}(n, m)$ star graph.

Definition $1.5\left(C_{3}(n, m, 1)\right.$ star graph). The graph obtained by attaching $n$ pendent vertices in one vertex of $C_{3}, m$ pendent vertices in other vertex of $C_{3}$ and 1 pendent vertices in remaining vertex of $C_{3}$ is called $C_{3}(n, m, 1)$ star graph. 


\section{Main Result}

Theorem 2.1. The star graph $G_{1, n}$ is fuzzy quotient-3 cordial.

Proof. Let $G$ be a Star graph $G_{1, n}$.

$V(G)=\{v\} \cup\left\{v_{k}: 1 \leq k \leq n\right\}$ and $E(G)=\left\{\left(v v_{k}\right): 1 \leq k \leq\right.$ $n\}$.

Here $|V(G)|=n+1,|E(G)|=n$.

Define $\sigma: V(G) \rightarrow[0,1]$ by $\sigma(v)=0.1, v$ is the central vertex of $G_{1, n}$

For the remaining vertices we have the following cases.

Case $\mathbf{i}:$ if $n \equiv 0(\bmod 3)$

$\begin{array}{ll}\sigma\left(v_{k}\right)=0.1 & 1 \leq k \leq \frac{n}{3} \\ \sigma\left(v_{k}\right)=0.2 & \frac{n}{3}+1 \leq k \leq \frac{2 n}{3} \\ \sigma\left(v_{k}\right)=0.3 & \frac{2 n}{3}+1 \leq k \leq n\end{array}$

Case ii : if $n \equiv 1(\bmod 3)$

$\sigma\left(v_{k}\right)=0.1 \quad 1 \leq k \leq \frac{n-1}{3}$

$\sigma\left(v_{k}\right)=0.2 \quad \frac{n-1}{3}+1 \leq k \leq \frac{2 n-2}{3}$

$\sigma\left(v_{k}\right)=0.3 \quad \frac{2 n-2}{3}+1 \leq k \leq n$

Case iii : if $n \equiv 2(\bmod 3)$

$\sigma\left(v_{k}\right)=0.1 \quad 1 \leq k \leq \frac{n-2}{3}$

$\sigma\left(v_{k}\right)=0.2 \quad \frac{n-2}{3}+1 \leq k \leq \frac{2 n-1}{3}$

$\sigma\left(v_{k}\right)=0.3 \quad \frac{2 n-1}{3}+1 \leq k \leq n$

For $n \equiv l(\bmod 3)$, where $0 \leq l \leq 2$, the following table shows that the number of vertices labeled with $i$ and the number of vertices labeled with $j$ differ by at most 1 , where $i, j \in\left\{\frac{r}{10}, r \in Z_{4}-\{0\}\right\}$.

\begin{tabular}{|c|c|c|c|}
\hline$l$ & $v_{\sigma}(0.1)$ & $v_{\sigma}(0.2)$ & $v_{\sigma}(0.3)$ \\
\hline 0 & $\frac{n}{3}+1$ & $\frac{n}{3}$ & $\frac{n}{3}$ \\
\hline 1 & $\frac{n-1}{3}+1$ & $\frac{n-1}{3}$ & $\frac{n-1}{3}+1$ \\
\hline 2 & $\frac{n+1}{3}$ & $\frac{n+1}{3}$ & $\frac{n+1}{3}$ \\
\hline
\end{tabular}

For $n \equiv l(\bmod 3)$, where $0 \leq l \leq 2$, the following table shows that the number of edges labeled with $i$ and the number of edges labeled with $j$ differ by at most 1 , where $i, j \in\left\{\frac{r}{10}, r \in\right.$ $\left.Z_{4}-\{0\}\right\}$.

\begin{tabular}{|c|c|c|c|}
\hline$l$ & $e_{\mu}(0.1)$ & $e_{\mu}(0.2)$ & $e_{\mu}(0.3)$ \\
\hline 0 & $\frac{n}{3}$ & $\frac{n}{3}$ & $\frac{n}{3}$ \\
\hline 1 & $\frac{n-1}{3}+1$ & $\frac{n-1}{3}$ & $\frac{n-1}{3}$ \\
\hline 2 & $\frac{n-2}{3}+1$ & $\frac{n-2}{3}+1$ & $\frac{n-2}{3}$ \\
\hline
\end{tabular}

From the above two tables it is concluded that the star graph $G_{1, n}$ is fuzzy quotient-3 cordial.

Example 2.2. Fuzzy quotient-3 Cordial labeling for the Star graph $G_{1,8}$

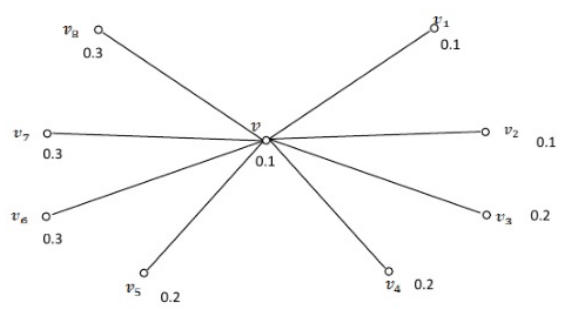

Figure 1

Theorem 2.3. The graph $C_{3}(n)$ is Fuzzy quotient-3 cordial.

Proof. Let $V(G)=\left\{u_{i}: 1 \leq i \leq 3\right\} \cup\left\{v_{k}: 1 \leq k \leq n\right\}$ and

$E(G)=\left\{\left[\left(u_{i} u_{i+1}\right) ; 1 \leq i \leq 2\right] \cup\left[\left(u_{3} v_{j}\right): 1 \leq j \leq n\right]\right\}$.

Here $|V(G)|=n+3,|E(G)|=n+3$.

Define $\sigma: V(G) \rightarrow[0,1]$ by $\sigma\left(u_{1}\right)=\sigma\left(u_{2}\right)=0.1$ and $\sigma\left(v_{1}\right)=$ $\sigma\left(v_{2}\right)=0.2, \sigma\left(v_{3}\right)=0.3$

For the remaining vertices we have following cases.

Case $\mathbf{i}:$ if $n \equiv 0(\bmod 3)$

$\sigma\left(v_{k+3}\right)=0.1 \quad 1 \leq k \leq \frac{n-3}{3}$

$\sigma\left(v_{k+3}\right)=0.2 \quad \frac{n-3}{3}+1 \leq k \leq \frac{2(n-3)}{3}$

$\sigma\left(v_{k+3}\right)=0.3 \quad \frac{2(n-3)}{3}+1 \leq k \leq n-3$

Case ii : if $n \equiv 1(\bmod 3)$

$\sigma\left(v_{k+3}\right)=0.1 \quad 1 \leq k \leq \frac{n-1}{3}$

$\sigma\left(v_{k+3}\right)=0.2 \quad \frac{n-1}{3}+1 \leq k \leq \frac{2 n-5}{3}$

$\sigma\left(v_{k+3}\right)=0.3 \quad \frac{2 n-5}{3}+1 \leq k \leq n-3$

Case iii : if $n \equiv 2(\bmod 3)$

$\sigma\left(v_{k+3}\right)=0.1 \quad 1 \leq k \leq \frac{n-2}{3}$

$\sigma\left(v_{k+3}\right)=0.2 \quad \frac{n-2}{3}+1 \leq k \leq \frac{2 n-4}{3}$

$\sigma\left(v_{k+3}\right)=0.3 \quad \frac{2 n-4}{3}+1 \leq k \leq n-3$

For $n \equiv l(\bmod 3)$, where $0 \leq l \leq 2$, the following table shows that the number of vertices labeled with $i$ and the number of vertices labeled with $j$ differ by at most 1 , where $i, j \in\left\{\frac{r}{10}, r \in Z_{4}-\{0\}\right\}$.

\begin{tabular}{|c|c|c|c|}
\hline$l$ & $v_{\sigma}(0.1)$ & $v_{\sigma}(0.2)$ & $v_{\sigma}(0.3)$ \\
\hline 0 & $\frac{n+3}{3}$ & $\frac{n+3}{3}$ & $\frac{n+3}{3}$ \\
\hline 1 & $\frac{n+2}{3}+1$ & $\frac{n+2}{3}$ & $\frac{n+2}{3}$ \\
\hline 2 & $\frac{n+1}{3}+1$ & $\frac{n+1}{3}+1$ & $\frac{n+1}{3}$ \\
\hline
\end{tabular}

For $n \equiv l(\bmod 3)$, where $0 \leq l \leq 2$, the following table shows that the number of edges labeled with $i$ and the number of edges labeled with $j$ differ by at most 1 , where $i, j \in\left\{\frac{r}{10}, r \in\right.$ $\left.Z_{4}-\{0\}\right\}$. 


\begin{tabular}{|c|c|c|c|}
\hline$l$ & $e_{\mu}(0.1)$ & $e_{\mu}(0.2)$ & $e_{\mu}(0.3)$ \\
\hline 0 & $\frac{n+3}{3}$ & $\frac{n+3}{3}$ & $\frac{n+3}{3}$ \\
\hline 1 & $\frac{n+2}{3}+1$ & $\frac{n+2}{3}$ & $\frac{n+2}{3}$ \\
\hline 2 & $\frac{n+1}{3}+1$ & $\frac{n+1}{3}+1$ & $\frac{n+1}{3}$ \\
\hline
\end{tabular}

From the above two tables it is concluded that the graph $C_{3}(n)$ is Fuzzy quotient-3 cordial.

Example 2.4. A graph obtained by attaching a star $G_{1,7}$ with any vertex of $C_{3}$ are Fuzzy quotient-3 cordial.

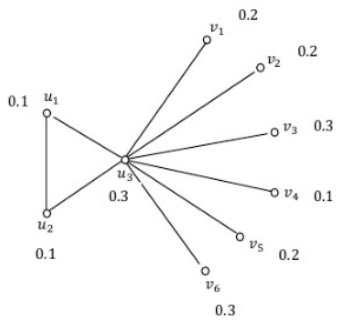

Figure 2

Theorem 2.5. The graph $C_{3}(n, n)$ is Fuzzy quotient-3 cordial.

Proof. Let $V(G)=\left\{u_{i}: 1 \leq i \leq 3\right\} \cup\left\{y_{k}: 1 \leq k \leq n\right\} \cup\left\{x_{j}\right.$ : $1 \leq j \leq n\}$ and

$E(G)=\left\{\left[\left(u_{i} u_{i+1}\right) ; 1 \leq i \leq 2\right] \cup\left[\left(u_{1} y_{k}\right): 1 \leq j \leq n\right] \cup\left[\left(u_{2} x_{j}\right):\right.\right.$ $1 \leq k \leq n]\}$

Here $|V(G)|=2 n+3,|E(G)|=2 n+3$.

Define $\sigma: V(G) \rightarrow[0,1]$ by $\sigma\left(u_{1}\right)=0.1, \sigma\left(u_{2}\right)=0.3$ and $\sigma\left(y_{1}\right)=\sigma\left(y_{2}\right)=0.2, \sigma\left(y_{3}\right)=0.1$

For the remaining vertices we have following cases.

Case $\mathbf{i}:$ if $n \equiv 0(\bmod 3)$

$$
\begin{array}{ll}
\sigma\left(y_{k+3}\right)=0.2 & 1 \leq k \leq \frac{n-3}{3} \\
\sigma\left(y_{k+3}\right)=0.1 & \frac{n-3}{3}+1 \leq k \leq \frac{2(n-3)}{3} \\
\sigma\left(y_{k+3}\right)=0.3 & \frac{2(n-3)}{3}+1 \leq k \leq n-3 \\
\sigma\left(x_{j}\right)=0.1 & 1 \leq j \leq \frac{n}{3} \\
\sigma\left(x_{j}\right)=0.3 & \frac{n}{3}+1 \leq j \leq \frac{2 n}{3} \\
\sigma\left(x_{j}\right)=0.2 & \frac{2 n}{3}+1 \leq j \leq n
\end{array}
$$

Case ii : if $n \equiv 1(\bmod 3)$

$$
\begin{array}{lc}
\sigma\left(y_{k+3}\right)=0.2 & 1 \leq k \leq \frac{n-1}{3} \\
\sigma\left(y_{k+3}\right)=0.1 & \frac{n-1}{3}+1 \leq k \leq \frac{2 n-5}{3} \\
\sigma\left(y_{k+3}\right)=0.3 & \frac{2 n-5}{3}+1 \leq k \leq n-3 \\
\sigma\left(x_{j}\right)=0.1 & 1 \leq j \leq \frac{n+2}{3} \\
\sigma\left(x_{j}\right)=0.3 & \frac{n+2}{3}+1 \leq j \leq \frac{2 n+1}{3} \\
\sigma\left(x_{j}\right)=0.2 & \frac{2 n+1}{3}+1 \leq j \leq n
\end{array}
$$

Case iii : if $n \equiv 2(\bmod 3)$

$$
\begin{array}{ll}
\sigma\left(y_{k+3}\right)=0.2 & 1 \leq k \leq \frac{n-2}{3} \\
\sigma\left(y_{k+3}\right)=0.1 & \frac{n-2}{3}+1 \leq k \leq \frac{2 n-4}{3}
\end{array}
$$

$$
\begin{array}{ll}
\sigma\left(y_{k+3}\right)=0.3 & \frac{2 n-4}{3}+1 \leq k \leq n-3 \\
\sigma\left(x_{j}\right)=0.1 & 1 \leq j \leq \frac{n+1}{3} \\
\sigma\left(x_{j}\right)=0.3 & \frac{n+1}{3}+1 \leq j \leq \frac{2 n+2}{3} \\
\sigma\left(x_{j}\right)=0.2 & \frac{2 n+2}{3}+1 \leq j \leq n
\end{array}
$$

For $n \equiv l(\bmod 3)$, where $0 \leq l \leq 2$, the following table shows that the number of vertices labeled with $i$ and the number of vertices labeled with $j$ differ by at most 1 , where $i, j \in\left\{\frac{r}{10}, r \in Z_{4}-\{0\}\right\}$.

\begin{tabular}{|l|c|c|c|}
\hline$l$ & $v_{\sigma}(0.1)$ & $v_{\sigma}(0.2)$ & $v_{\sigma}(0.3)$ \\
\hline 0 & $\frac{2 n+3}{3}$ & $\frac{2 n+3}{3}$ & $\frac{2 n+3}{3}$ \\
\hline 1 & $\frac{2 n+1}{3}+1$ & $\frac{2 n+1}{3}+1$ & $\frac{2 n+1}{3}$ \\
\hline 2 & $\frac{2 n+2}{3}+1$ & $\frac{2 n+2}{3}$ & $\frac{2 n+2}{3}$ \\
\hline
\end{tabular}

For $n \equiv l(\bmod 3)$, where $0 \leq l \leq 2$, the following table shows that the number of edges labeled with $i$ and the number of edges labeled with $j$ differ by at most 1 , where $i, j \in\left\{\frac{r}{10}, r \in\right.$ $\left.Z_{4}-\{0\}\right\}$.

\begin{tabular}{|c|c|c|c|}
\hline$l$ & $e_{\mu}(0.1)$ & $e_{\mu}(0.2)$ & $e_{\mu}(0.3)$ \\
\hline 0 & $\frac{2 n+3}{3}$ & $\frac{2 n+3}{3}$ & $\frac{2 n+3}{3}$ \\
\hline 1 & $\frac{2 n+1}{3}+1$ & $\frac{2 n+1}{3}+1$ & $\frac{2 n+1}{3}$ \\
\hline 2 & $\frac{2 n+2}{3}$ & $\frac{2 n+2}{3}$ & $\frac{2 n+2}{3}+1$ \\
\hline
\end{tabular}

From the above two tables it is concluded that the graph $C_{3}(n, n)$ is Fuzzy quotient- 3 cordial.

Theorem 2.6. The graph $C_{3}(n, n, n)$ is Fuzzy quotient-3 cordial.

Proof. Let $V(G)=\left\{u_{i}: 1 \leq i \leq 3\right\} \cup\left\{y_{k}: 1 \leq k \leq n\right\} \cup\left\{x_{j}\right.$ : $1 \leq j \leq n\} \cup\left\{z_{r}: 1 \leq r \leq n\right\}$ and

$E(G)=\left\{\left[\left(u_{i} u_{i+1}\right) ; 1 \leq i \leq 2\right] \cup\left[\left(u_{1} y_{k}\right): 1 \leq j \leq n\right] \cup\left[\left(u_{2} x_{j}\right):\right.\right.$ $\left.1 \leq k \leq n] \cup\left[\left(u_{3} z_{r}\right): 1 \leq r \leq n\right]\right\}$

Here $|V(G)|=3 n+3,|E(G)|=3 n+3$.

Define $\sigma: V(G) \rightarrow[0,1]$ by $\sigma\left(u_{1}\right)=0.1, \sigma\left(u_{2}\right)=0.3, \sigma\left(u_{3}\right)=$ 0.3 and $\sigma\left(y_{1}\right)=\sigma\left(y_{2}\right)=0.2, \sigma\left(y_{3}\right)=0.1$

For the remaining vertices we have following cases.

Case $\mathbf{i}:$ if $n \equiv 0(\bmod 3)$

$$
\begin{array}{ll}
\sigma\left(y_{k+3}\right)=0.2 & 1 \leq k \leq \frac{n-3}{3} \\
\sigma\left(y_{k+3}\right)=0.1 & \frac{n-3}{3}+1 \leq k \leq \frac{2(n-3)}{3} \\
\sigma\left(y_{k+3}\right)=0.3 & \frac{2(n-3)}{3}+1 \leq k \leq n-3 \\
\sigma\left(x_{j}\right)=0.1 & 1 \leq j \leq \frac{n}{3} \\
\sigma\left(x_{j}\right)=0.3 & \frac{n}{3}+1 \leq j \leq \frac{2 n}{3} \\
\sigma\left(x_{j}\right)=0.2 & \frac{2 n}{3}+1 \leq j \leq n \\
\sigma\left(z_{r}\right)=0.1 & 1 \leq r \leq \frac{n}{3} \\
\sigma\left(z_{r}\right)=0.3 & \frac{n}{3}+1 \leq j \leq \frac{2 n}{3} \\
\sigma\left(z_{r}\right)=0.2 & \frac{2 n}{3}+1 \leq j \leq n
\end{array}
$$

Case ii : if $n \equiv 1(\bmod 3)$ 


$$
\begin{array}{ll}
\sigma\left(y_{k+3}\right)=0.2 & 1 \leq k \leq \frac{n-1}{3} \\
\sigma\left(y_{k+3}\right)=0.1 & \frac{n-1}{3}+1 \leq k \leq \frac{2 n-5}{3} \\
\sigma\left(y_{k+3}\right)=0.3 & \frac{2 n-5}{3}+1 \leq k \leq n-3 \\
\sigma\left(x_{j}\right)=0.1 & 1 \leq j \leq \frac{n+2}{3} \\
\sigma\left(x_{j}\right)=0.3 & \frac{n+2}{3}+1 \leq j \leq \frac{2 n+1}{3} \\
\sigma\left(x_{j}\right)=0.2 & \frac{2 n+1}{3}+1 \leq j \leq n \\
\sigma\left(z_{r}\right)=0.1 & 1 \leq r \leq \frac{n-1}{3} \\
\sigma\left(z_{r}\right)=0.3 & \frac{n-1}{3}+1 \leq j \leq \frac{2 n+1}{3} \\
\sigma\left(z_{r}\right)=0.2 & \frac{2 n+1}{3}+1 \leq j \leq n
\end{array}
$$

Case iii : if $n \equiv 2(\bmod 3)$

$$
\begin{array}{ll}
\sigma\left(y_{k+3}\right)=0.2 & 1 \leq k \leq \frac{n+1}{3} \\
\sigma\left(y_{k+3}\right)=0.1 & \frac{n+1}{3}+1 \leq k \leq \frac{2 n-4}{3} \\
\sigma\left(y_{k+3}\right)=0.3 & \frac{2 n-4}{3}+1 \leq k \leq n-3 \\
\sigma\left(x_{j}\right)=0.1 & 1 \leq j \leq \frac{n+1}{3} \\
\sigma\left(x_{j}\right)=0.3 & \frac{n+1}{3}+1 \leq j \leq \frac{2 n+2}{3} \\
\sigma\left(x_{j}\right)=0.2 & \frac{2 n+2}{3}+1 \leq j \leq n \\
\sigma\left(z_{r}\right)=0.1 & 1 \leq r \leq \frac{n+1}{3} \\
\sigma\left(z_{r}\right)=0.3 & \frac{n+1}{3}+1 \leq r \leq \frac{2 n+2}{3} \\
\sigma\left(z_{r}\right)=0.2 & \frac{2 n+2}{3}+1 \leq r \leq n
\end{array}
$$

For $n \equiv l(\bmod 3)$, where $0 \leq l \leq 2$, the following table shows that the number of vertices labeled with $i$ and the number of vertices labeled with $j$ differ by at most 1 , where $i, j \in\left\{\frac{r}{10}, r \in Z_{4}-\{0\}\right\}$.

\begin{tabular}{|c|c|c|c|}
\hline$l$ & $v_{\sigma}(0.1)$ & $v_{\sigma}(0.2)$ & $v_{\sigma}(0.3)$ \\
\hline 0 & $n+1$ & $n+1$ & $n+1$ \\
\hline 1 & $n+1$ & $n+1$ & $n+1$ \\
\hline 2 & $n+1$ & $n+1$ & $n+1$ \\
\hline
\end{tabular}

For $n \equiv l(\bmod 3)$, where $0 \leq l \leq 2$, the following table shows that the number of edges labeled with $i$ and the number of edges labeled with $j$ differ by at most 1 , where $i, j \in\left\{\frac{r}{10}, r \in\right.$ $\left.Z_{4}-\{0\}\right\}$.

\begin{tabular}{|c|c|c|c|}
\hline$l$ & $e_{\mu}(0.1)$ & $e_{\mu}(0.2)$ & $e_{\mu}(0.3)$ \\
\hline 0 & $n+1$ & $n+1$ & $n+1$ \\
\hline 1 & $n+1$ & $n+1$ & $n+1$ \\
\hline 2 & $\frac{2 n+2}{3}$ & $\frac{2 n+2}{3}$ & $\frac{2 n+2}{3}+1$ \\
\hline
\end{tabular}

From the above two tables it is concluded that the graph $C_{3}(n, n, n)$ is Fuzzy quotient-3 cordial.

Example 2.7. A graph obtained by attaching three copies of a star $G_{1,3}$ with each vertices of $C_{3}$ are Fuzzy quotient-3 cordial.

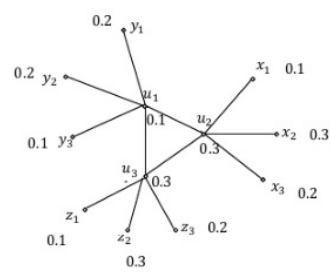

Figure 3

\section{Conclusion}

In this paper, it is proved that some star related graphs which admits Fuzzy quotient-3 cordial. The existence of Fuzzy quotient- 3 cordial labeling of different families of graphs will be the future work.

\section{References}

[1] Joseph A. Gallian, A Dynamic survey of Graph Labeling, Nineteenth Edition, 2016.

[2] I. Cahit, Cordial Graphs: A weaker version of Graceful and Harmonious graphs, Ars combin., 23(1987), 201-207.

[3] P. Sumathi, A. Mahalakshmi and A. Rathi, Quotient-3 Cordial Labeling for Star Related Graphs, Global Journal of Pure and Applied Mathematics, 13(7)(2017), 39093918.

[4] P. Sumathi, A. Mahalakshmi and A. Rathi, Quotient-3 Cordial Labeling for cycle related graphs, International journal of innovative Research in Applied Sciences and Engineering, 1(2)(2017), 12-19.

[5] P. Sumathi, A. Mahalakshmi and A. Rathi, Quotient-3 Cordial Labeling for path related graphs part-I, International Journal of Pure and Applied Mathematics, 115(9)(2017), 249-258.

[6] P. Sumathi, A. Mahalakshmi and A. Rathi, Quotient-3 cordial labeling for some Unicyclic graphs-Part-II, International of Journal Innovative Research in Pure and Engineering Mathematics (IJIRPEM), 1(1)(2017), 9-16.

$\operatorname{ISSN}(\mathrm{P}): 2319-3786$

Malaya Journal of Matematik $\operatorname{ISSN}(\mathrm{O}): 2321-5666$

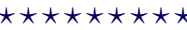

